\title{
NEUTRINO PHYSICS: OPEN THEORETICAL QUESTIONS
}

\author{
A. Y. SMIRNOV \\ International Centre for Theoretical Physics, Strada Costiera 11, 31014 Trieste, Italy \\ and \\ Institute for Nuclear Research, Russian Academy of Sciences,Moscow, Russia \\ E-mail: smirnov@ictp.trieste.it
}

\begin{abstract}
We know that neutrino mass and mixing provide a window to physics beyond the Standard Model. Now this window is open, at least partly. And the questions are: what do we see, which kind of new physics, and how far "beyond"? I summarize the present knowledge of neutrino mass and mixing, and then formulate the main open questions. Following the bottom-up approach, properties of the neutrino mass matrix are considered. Then different possible ways to uncover the underlying physics are discussed. Some results along the line of: see-saw, GUT and SUSY GUT are reviewed.
\end{abstract}

\section{Introduction}

This review ${ }^{a}$ is devoted to neutrino masses and mixing. It covers experimental results, their interpretation and implications. It is in this area that enormous progress has been achieved during the last few years.

The field develops fast, and already after the Symposium a number of important results have been published including the SNO salt phase data, new analysis of the Heidelberg-Moscow experimental results, etc..

In Sec. 2 the main achievements in reconstruction of the neutrino mass and mixing spectrum are summarized. The open theoretical questions are formulated in Sec. 3. In Sec. 4, following the bottomup approach, the neutrino mass matrix is reconstructed and its properties are studied. In Sec. 5 the ways we may go in answering the open questions are outlined.

\section{What Have We Learned?}

\subsection{Solar Neutrinos}

The latest SNO salt phase results ${ }^{1}$ have further confirmed the correctness of the Standard Solar Model (SSM) neutrino fluxes ${ }^{2}$ and the realization of the MSW large mixing (LMA) conversion mechanism ${ }^{3}$ inside the Sun. ${ }^{4-10}$ In Fig. 1 we show the allowed region of the oscillation parameters $\tan ^{2} \theta_{12}$ and $\Delta m_{12}^{2}$ from the $2 \nu$ combined analysis of the solar neutrino

${ }^{a}$ Talk given at the XXI International Symposium on Lepton and Photon Interactions at High Energies, "Lepton Photon 2003", August 11-16, 2003 - Fermilab, Batavia, IL USA. and $\mathrm{KamLAND}^{11}$ results. The best-fit values of the parameters are

$$
\Delta m_{12}^{2}=7.1 \times 10^{-5} \mathrm{eV}^{2}, \quad \tan ^{2} \theta_{12}=0.4 .
$$

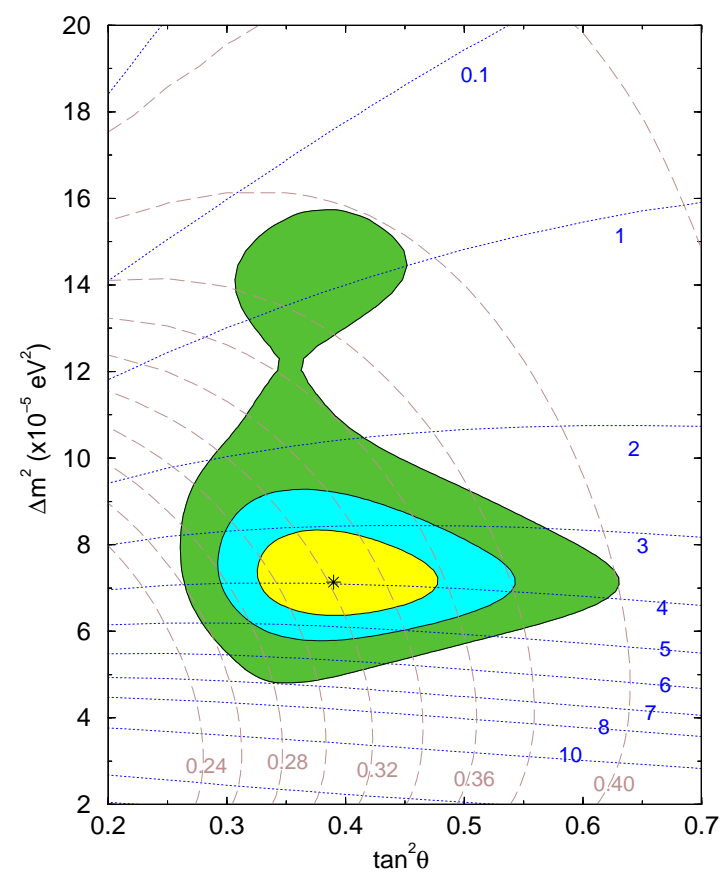

Figure 1. The allowed regions of oscillation parameters from the combined fit of the solar neutrino data and the KamLAND spectrum at $1 \sigma, 2 \sigma, 3 \sigma$ CL. ${ }^{10}$ Shown are also the contours of constant $\mathrm{CC} / \mathrm{NC}$ ratio (dotted lines) and the Day-Night asymmetry (dashed lines) at SNO (numbers on the curves in $\%)$.

Combined fit of the solar, KamLAND ${ }^{11}$ and CHOOZ $^{12}$ results favors nearly zero 1-3 mixing: $\sin ^{2} \theta_{13} \sim 0{ }^{9,10}$ Basically the data have selected the l-LMA region with $\Delta m_{12}^{2}<10^{-4} \mathrm{eV}^{2}$ (the h-LMA 
region is accepted now at $3 \sigma$ only), and strongly disfavored maximal 1-2 mixing. The upper bound is

$$
\tan ^{2} \theta_{12}<0.64 \quad(3 \sigma) .
$$

As a result of these improvements, the physics of the conversion is now even determined quantitatively. 5,10 In particular, recent results show relevance of the notion of resonance, they fix the relative strength of the effects of the adiabatic conversion and the oscillations as function of the neutrino energy. ${ }^{10}$

In Fig. 1 we show also the contours of constant $\mathrm{CC} / \mathrm{NC}$ ratio and Day-Night asymmetry of the CCevents at SNO. They allow one to evaluate an impact of future SNO measurements. The KamLAND operation will allow one to eventually determine $\Delta m_{12}^{2}$ with about $10 \%$ accuracy.

Are there any data which indicate deviation from the LMA picture? In this connection we consider two generic features of the LMA-MSW solution:

- the predicted Ar-production rate, $Q_{A r}=$ $2.96 \pm 0.25 \mathrm{SNU}$, is about $2 \sigma$ higher than the Homestake $^{13}$ result; and

- the upturn of the spectrum at low energies, that is, the increase of the ratio $N^{o b s} / N^{S S M}$ with decrease of energy, is expected which can be as large as $10-15 \%$. However, the latest SNO as well as the previous SNO and SuperKamiokande ${ }^{14}$ spectral data do not show the upturn, being in agreement with the absence of distortion.

Both problems can be resolved simultaneously, if a light sterile neutrino exists with very small activesterile mixing: ${ }^{15}$

$$
\frac{\Delta m_{01}^{2}}{\Delta m_{21}^{2}}=0.05-0.2, \quad \sin ^{2} 2 \alpha=10^{-5}-10^{-3} \text {. }
$$

Such a mixing produces a dip in the survival probability (Fig. 2) which suppresses both the Arproduction rate and the upturn of spectrum. The best description of the data would correspond to the dip at relatively high energies (panel for $R_{\Delta}=0.10$ ) when the CNO- and pep-neutrino fluxes and the low energy part of the boron neutrino spectrum are suppressed.

Such a possibility can be tested in the future low energy neutrino experiments: BOREXINO, ${ }^{16}$ KamLAND, MOON, etc. ${ }^{17}$ as well as in further measurements of the spectrum by SNO and SK.

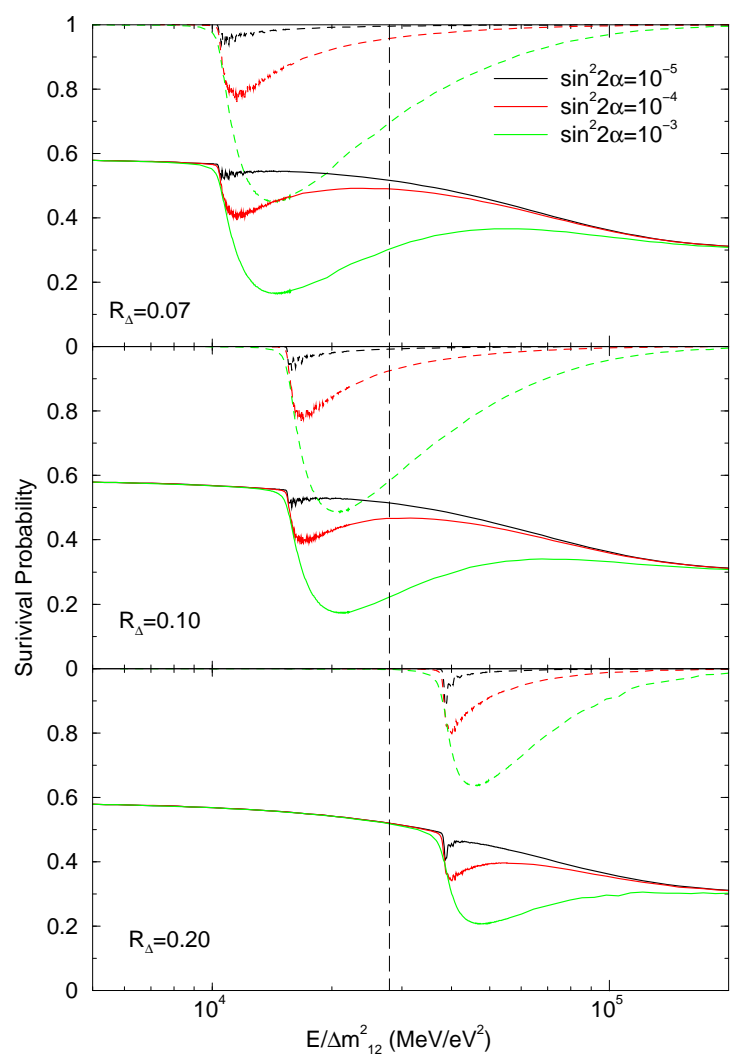

Figure 2. The survival probabilities of the electron neutrino (solid line) and the active neutrinos (dashed line) for different values of the sterile-active mixing. ${ }^{15}$ The panels correspond to three different values of $R_{\Delta} \equiv \Delta m_{01}^{2} / \Delta m_{21}^{2}$. Vertical dashed line indicates the position of the 1-2 resonance.

\subsection{Atmospheric Neutrinos}

A recent refined analysis of the SuperKamiokande data in terms of $\nu_{\mu}-\nu_{\tau}$ oscillations gives ${ }^{18}$ at $90 \%$ C.L.

$$
\Delta m_{13}^{2}=(1.3-3.0) \times 10^{-3} \mathrm{eV}^{2}, \quad \sin ^{2} 2 \theta_{23}>0.9
$$

with the best fit at $\Delta m_{12}^{2}=2.0 \times 10^{-3} \mathrm{eV}^{2}$ and $\sin ^{2} 2 \theta_{12}=1.0$. Combined analysis of the CHOOZ and the atmospheric neutrino data puts the upper bound on the 1-3 mixing ${ }^{19}$

$$
\sin ^{2} \theta_{13}<0.067 \quad(3 \sigma) .
$$

The open question is whether oscillations of the atmospheric $\nu_{e}$ exist? There are two possible sources of these oscillations: (i) non-zero 1-3 mixing and "atmospheric" $\Delta m_{13}^{2}$, and (ii) solar oscillation parameters in Eq. (1). Also their interference should exist. ${ }^{20}$ After confirmation of the LMA-MSW solution we can definitely say that oscillations driven by the LMA parameters (the LMA oscillations) should show up at 
some level. Relative modification of the $\nu_{e}$ flux due to the LMA oscillations can be written $\mathrm{as}^{20}$

$$
\frac{F_{e}}{F_{e}^{0}}-1=P_{2}\left(r \cos ^{2} \theta_{23}-1\right),
$$

where $P_{2}\left(\Delta m_{12}^{2}, \theta_{12}\right)$ is the $2 \nu$ transition probability and $r \equiv F_{\mu}^{0} / F_{e}^{0}$ is the ratio of the original $\nu_{\mu}$ and $\nu_{e}$ fluxes. In the sub-GeV region, where $P_{2}$ can be of the order 1 , the ratio equals $r \approx 2$, so that the oscillation effect is proportional to the deviation of the 2-3 mixing from the maximal value: $D_{23} \equiv 1 / 2-\sin ^{2} \theta_{23}$. In Fig. 3 we show the ratio of numbers of the $e$-like events with and without oscillations as function of the zenith angle of the electron. For the allowed range of $\sin ^{2} \theta_{23}$ and the present best-fit value of $\Delta m_{12}^{2}$ the excess can be as large as $5-6 \%$. The excess increases with decreasing energy.

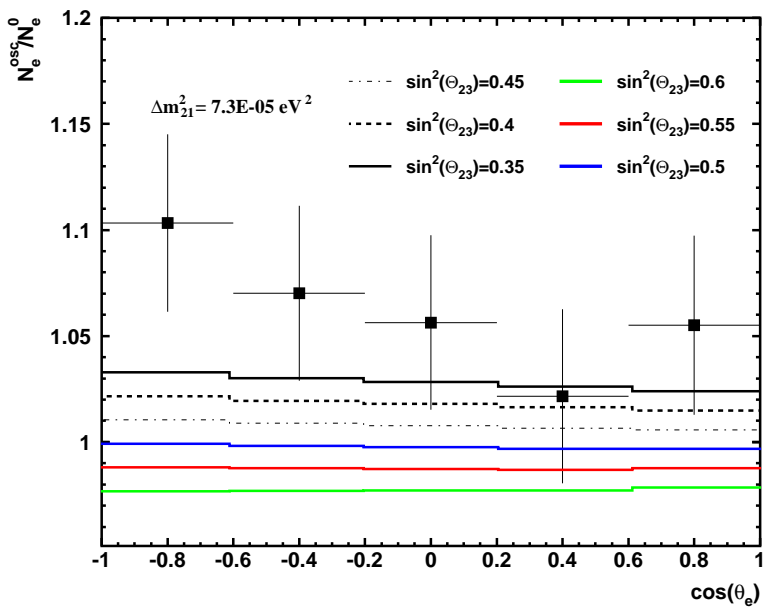

Figure 3. The ratio of numbers of the $e$-like events with and without oscillations as function of the zenith angle of the electron for different values of $\sin ^{2} \theta_{23} .{ }^{20}$ Other parameters are $\sin ^{2} 2 \theta_{12}=0.82, \sin \theta_{13}=0$ and $\Delta m_{12}^{2}=7.3 \times 10^{-5} \mathrm{eV}^{2}$. Also shown are the SuperKamiokande experimental points.

Future searches for the excess can be used to restrict or measure $D_{23}$. In fact, the latest analysis, (without renormalization of the original fluxes) shows some excess of the $e$-like events at low energies and the absence of excess in the multi-GeV sample, thus giving a hint of non-zero $D_{23}$. Establishing this deviation has important consequences for understanding the origins of neutrino masses and mixing.

Non-zero 1-3 mixing generates the interference effect between the LMA oscillations amplitudes. ${ }^{20}$ The interference contribution does not contain the "screening" factor, in Eq. (6), and can reach $2-4 \%$ for the allowed values of $\sin \theta_{13}$. This produces an uncertainty in the determination of $D_{23}$. So, $D_{23}$ can be measured if either a large excess is found or/and a stronger bound on the 1-3 mixing is established.

\subsection{Mass Spectrum and Mixing}

Information obtained from the oscillation experiments allows us to make significant progress in the reconstruction of the neutrino mass and flavor spectrum (Fig. 4).
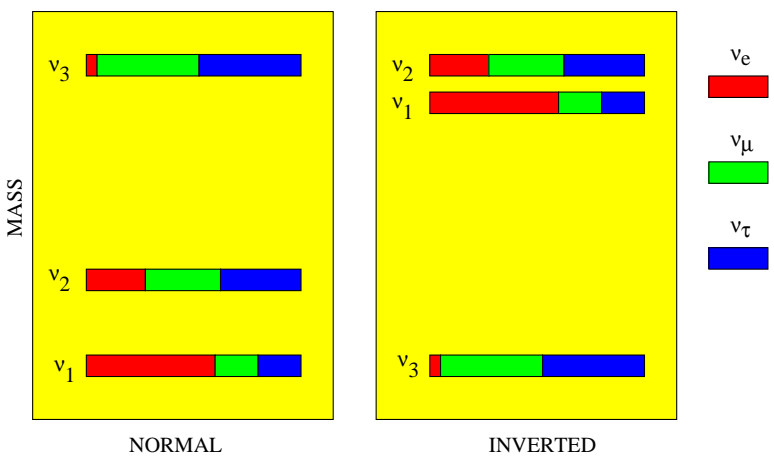

Figure 4. Neutrino mass and flavor spectra for the normal (left) and inverted (right) mass hierarchies. The distribution of flavors (colored parts of boxes) in the mass eigenstates corresponds to the best-fit values of mixing parameters and $\sin ^{2} \theta_{13}=0.05$.

The unknowns are:

(i) admixture of $\nu_{e}$ in $\nu_{3}: U_{e 3}$;

(ii) type of mass spectrum: hierarchical; nonhierarchical with certain ordering; degenerate, which is related to the value of the absolute mass scale, $m_{1}$; and

(iii) type of mass hierarchy (ordering): normal, inverted.

Using a global fit of the oscillation data one can find intervals for the elements of the PMNS mixing matrix $\left\|U_{\alpha i}\right\|$ :

$$
\left(\begin{array}{ccc}
0.79-0.86 & 0.50-0.61 & 0.0-0.16 \\
0.24-0.52 & 0.44-0.69 & 0.63-0.79 \\
0.26-0.52 & 0.47-0.71 & 0.60-0.77
\end{array}\right),
$$

where columns correspond to the flavor index and rows to the mass index. ${ }^{21}$

Now we are in a position to construct the leptonic unitarity triangle, although the finite size of one angle is still unknown. For practical reason (no intensive $\nu_{\tau}$ beams) we consider the triangle which employs the $e$ - and $\mu$ - rows of the mixing matrix (Fig. 5). The triangle is not degenerate in spite of the strong bound on the 1-3 mixing. 
Is it possible to reconstruct the triangle using results from future experiments? Can we use the triangle to determine the CP-violation phase, $\delta$ ? The area of the triangle is related to the Jarlskog invariant $J_{C P} \equiv \operatorname{Im}\left[U_{e 1} U_{\mu 2} U_{e 2}^{*} U_{\mu 1}^{*}\right]: S=J_{C P} / 2$. Reconstruction of the triangle is complementary to measurements of the neutrino-antineutrino asymmetries in oscillations. Interestingly, the main problem here is the coherence: the same coherence which leads to the oscillations. For the triangle method we need to study interactions of the mass eigenstates, whereas in practice we deal with flavor (coherent) states. So, breaking of the coherence, averaging of oscillations, experiments with the beams of mass eigenstates and measurements of the survival (rather than transition) probabilities are the key elements of the method. ${ }^{22}$

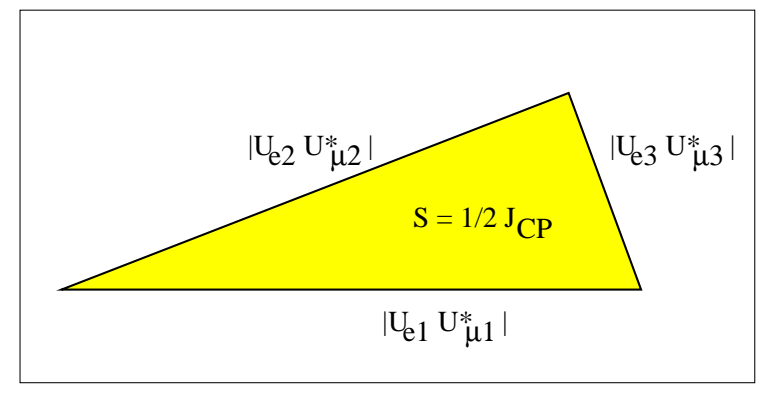

Figure 5. Possible leptonic unitarity triangle. We take the best-fit values of $\theta_{12}$, and $\theta_{23}$ and $\sin \theta_{13}=0.16$.

\subsection{Neutrinos from SN1987A}

After confirmation of the LMA-MSW solution we can definitely say that the effect of flavor conversion has already been observed in 1987. One must take into account the conversion effects in analysis of SN1987 $\mathrm{A}^{23}$ and future supernova neutrino data.

In terms of the original fluxes of the electron and muon antineutrinos, $F^{0}\left(\bar{\nu}_{e}\right)$ and $F^{0}\left(\bar{\nu}_{\mu}\right)$, the electron antineutrino flux at the detector can be written as

$$
F\left(\bar{\nu}_{e}\right)=F^{0}\left(\bar{\nu}_{e}\right)+\bar{p} \Delta F^{0},
$$

where $\Delta F^{0} \equiv F\left(\bar{\nu}_{\mu}\right)-F\left(\bar{\nu}_{e}\right)$, and $\bar{p}$ is the permutation factor. In assumptions of the normal mass hierarchy (ordering) and the absence of new neutrino states, $\bar{p}$ can be calculated precisely: $\bar{p}=1-P_{1 e}$, where $P_{1 e}$ is the probability of $\bar{\nu}_{1} \rightarrow \bar{\nu}_{e}$ transition inside the Earth. ${ }^{24,25}$ It can be written as $\bar{p}=$ $\sin ^{2} \theta_{12}+f_{\text {reg }}$, where $f_{\text {reg }}$ describes the effect of oscillations (regeneration of the $\bar{\nu}_{e}$ flux) inside the Earth.
Due to the difference in distances traveled by neutrinos to Kamiokande, IMB and Baksan detectors inside the Earth: $4363 \mathrm{~km}, 8535 \mathrm{~km}$ and $10449 \mathrm{~km}$ correspondingly, the permutation factors differ for these detectors (Fig. 6). The Earth matter effect can partially explain the difference between the Kamiokande and the IMB spectra of events. ${ }^{25}$

In contrast to $\bar{p}$, the original fluxes, and consequently $\Delta F^{0}$, are not well known, and one can not make precise predictions of the flux modification in Eq. (8).

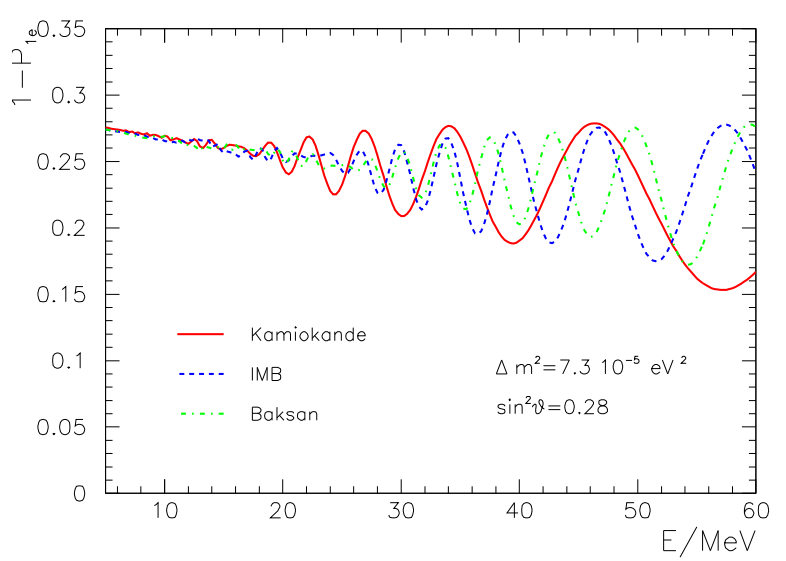

Figure 6. The permutation factor $\bar{p}=1-P_{1 e}$ as a function of neutrino energy for Kamiokande II, IMB and Baksan detectors. $^{26}$

For the inverted mass hierarchy and $\sin ^{2} \theta_{13}>$ $10^{-5}$ one would get a stronger permutation, $\bar{p}=1$, and therefore a harder $\bar{\nu}_{e}$ spectrum, as well as the absence of the Earth matter effect. This is disfavored by the data, ${ }^{27}$ though in view of small statistics and uncertainties in the original fluxes it is not possible to make a firm statement.

\subsection{Absolute Scale of Mass}

From the oscillation results we can put only a lower limit on the heaviest neutrino mass:

$$
m_{h} \geq \sqrt{\Delta m_{13}^{2}}>0.04 \mathrm{eV}
$$

where $m_{h}=m_{3}$ for the normal mass hierarchy, and $m_{h}=m_{1} \approx m_{2}$ for the inverted hierarchy. The neutrinoless double beta decay is determined by the combination

$$
m_{e e}=\left|\sum_{k} U_{e k}^{2} m_{k} e^{i \phi(k)}\right|,
$$

where $\phi(k)$ is the phase of the $k$ eigenvalue. Figure 7 summarizes the present knowledge of the absolute 
mass scale. Shown are the allowed regions in the plane of $m_{e e}$ probed by $\beta \beta_{0 \nu}$ decay and the mass of lightest neutrino probed by the direct kinematical methods and cosmology. The best present bound on $m_{e e}$ is given by the Heidelberg-Moscow experiment: $m_{e e}<0.35-0.50 \mathrm{eV},{ }^{29}$ part of collaboration claims evidence of a positive signal. ${ }^{30}$ Interestingly,

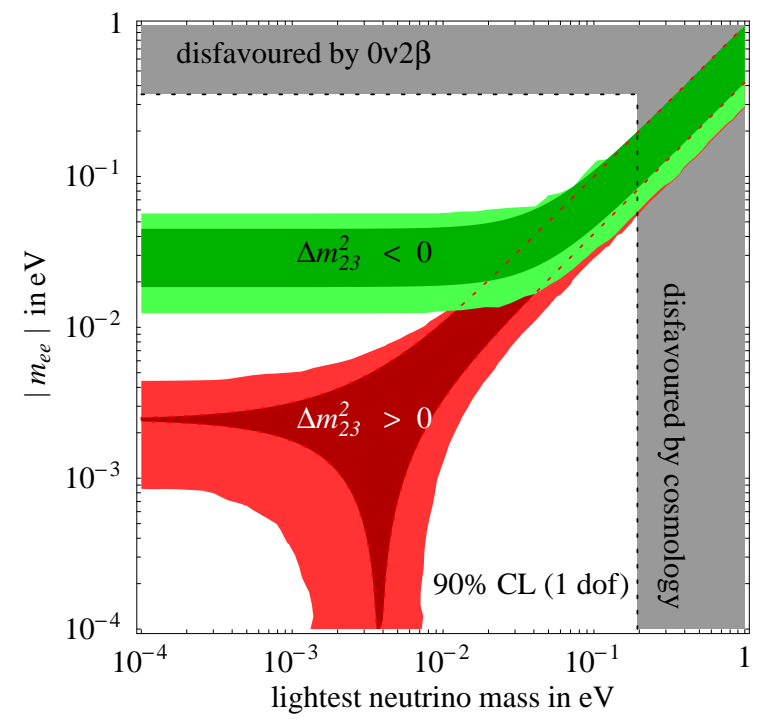

Figure 7. The $90 \%$ CL range for $m_{e e}$ as a function of the lightest neutrino mass for the normal $\left(\Delta m_{23}^{2}>0\right)$ and inverted $\left(\Delta m_{23}^{2}<0\right)$ mass hierarchies. ${ }^{28}$ The darker regions show how the allowed range for the present best-fit values of the parameters with negligible errors.

the present double beta decay measurements and cosmology have similar sensitivities $m_{e e} \sim m_{1} \sim$ $(0.2-0.5) \mathrm{eV}$. The latter corresponds to the degenerate mass spectrum: $m_{1} \approx m_{2} \approx m_{3} \equiv m_{0}$. Analyses of cosmological data (with WMAP) result in the $95 \%$ C.L. upper bounds $m_{0}<0.23 \mathrm{eV},{ }^{31} m_{0}<0.6 \mathrm{eV}^{32}$ and $m_{0}<0.34 \mathrm{eV} .^{33}$ Independent analysis which includes the $\mathrm{X}$-ray galaxy cluster data gives non-zero value $m_{0}=0.20 \pm 0.10 \mathrm{eV}^{34}$

Future improvements of the upper bound on $m_{e e}$ have the potential to distinguish between the hierarchies: According to Fig. 7, if the bound $m_{e e}<0.012$ $\mathrm{eV}$ is established, the inverted hierarchy will be excluded at $90 \%$ C.L..

\subsection{LSND}

The situation with this ultimate neutrino anomaly ${ }^{35}$ is really dramatic: all suggested physical (not related to the LSND methods) solutions are strongly or very strongly disfavored now. At the same time, being confirmed, the oscillation interpretation of the LSND result may change our understanding the neutrino (and in general fermion) masses.

A recent analysis performed by the KARMEN collaboration ${ }^{36}$ has further disfavored a scenario ${ }^{37}$ in which the $\bar{\nu}_{e}$ appearance is explained by the anomalous muon decay $\mu^{+} \rightarrow \bar{\nu}_{e} \bar{\nu}_{i} e^{+}(i=e, \mu, \tau)$.

The CPT-violation scheme ${ }^{38}$ with different mass spectra of neutrinos and antineutrinos is disfavored by the atmospheric neutrino data. ${ }^{39}$ No compatibility of LSND and "all but LSND" data have been found below $3 \sigma{ }^{40}$

The main problem of the $(3+1)$ scheme with $\Delta m^{2} \sim 1 \mathrm{eV}^{2}$ is that the predicted LSND signal, which is consistent with the results of other short base-line experiments (BUGEY, CHOOZ, CDHS, CCFR, KARMEN) as well as the atmospheric neutrino data, is too small: the $\bar{\nu}_{\mu} \rightarrow \bar{\nu}_{e}$ probability is about $3 \sigma$ below the LSND measurement.

Introduction of the second sterile neutrino with $\Delta m^{2}>8 \mathrm{eV}^{2}$ may help. ${ }^{41}$ It was shown ${ }^{42}$ that a new neutrino with $\Delta m^{2} \sim 22 \mathrm{eV}^{2}$ and mixings $U_{e 5}=0.06, U_{\mu 5}=0.24$ can enhance the predicted LSND signal by $(60-70) \%$. The $(3+2)$ scheme has, however, problems with cosmology and astrophysics.

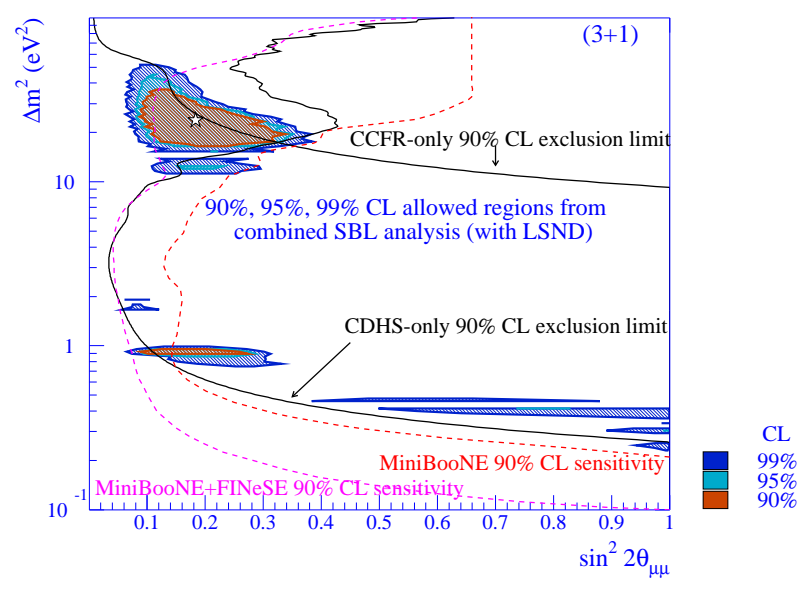

Figure 8. The allowed regions of parameters of the $(3+1)$ scheme, $\Delta m_{14}^{2}$ and $\sin ^{2} 2 \theta_{\mu \mu} \approx 4\left|U_{\mu 4}\right|^{2}$, at different confidence levels. Shown are the $90 \%$ sensitivity limits of the MiniBooNE and the MiniBooNE+FINeSE experiments.

The generic prediction of $(3+\mathrm{n})$ schemes is the $\nu_{\mu}$ oscillation disappearance at the level of ex- 
isting upper bouds from CDHS, ${ }^{43} \mathrm{CCFR},{ }^{44}$ and NOMAD ${ }^{45}$ experiments. New searches of $\nu_{\mu}$ disappearance are being performed by the MiniBooNE experiment ${ }^{46}$ and planned by the proposed experiment FINeSE $^{47}$ (see Fig. 8, where the sensitivity region of these searches is shown ${ }^{47}$ ).

The combination of the two described solutions, namely the $3+1$ scheme with CPT-violation, has been considered ${ }^{48}$

\subsection{Known and Unknown}

Information described in the previous sections can be summarized in the following way.

1. The observed ratio of the mass squared differences, $\Delta m_{12}^{2} / \Delta m_{23}^{2}=0.01-0.15$, implies that there is no strong hierarchy of masses:

$$
\frac{m_{2}}{m_{3}}>\sqrt{\frac{\Delta m_{12}^{2}}{\Delta m_{23}^{2}}}=0.18_{-0.08}^{+0.22} .
$$

For charge leptons the corresponding ratio is 0.06 .

2 . There is the bi-large or large-maximal mixing between the neighboring families $(1-2)$ and $(2-3)$. Still rather significant deviation of the 2-3 mixing from the maximal one is possible.

3. Mixing between remote (1-3) families is weak.

Several key elements are unknown yet leading to a variety of possible interpretations.

Knowledge of the absolute mass scale, type of mass spectrum, and type of mass hierarchy is of the highest priority. The 1-3 mixing has important phenomenological consequences; its value is a test of the mechanisms of the lepton mixing enhancement. The $\mathrm{CP}$-violating Majorana phases are extremely important for the structure of neutrino mass matrices. Deviations of the 2-3 and 1-2 mixings from maximal values play a crucial role in understanding the origins of neutrino masses. The existence of new neutrino states (their search should be a permanent item in the scientific agenda) may change completely our approaches to the underlying theory.

These are phenomenological and experimental questions we will deal with during the next 20-30 years.

\section{Open Theoretical Questions}

What does all this (results on neutrino masses and mixing) mean?
Among old, still open, questions are the following: Why are neutrino masses so small in comparison with the charged lepton and quark masses? What is the origin of neutrino mass? Is it the same as the one for quarks and charged leptons?

What are the relations between neutrino masses and other mass/energy scales in nature, e.g. the scale of cosmological constant or dark energy?

Why is the lepton mixing large? Why is it so different from quark mixing? Is the 2-3 mixing exactly maximal? What are the relations between different mixing angles (if any)? How is the observed pattern of lepton mixing is generated?

In the quark sector the smallness of mixing is related to the strong mass hierarchy. What are the relations between the lepton masses and lepton mixing?

Do neutrinos show certain flavor or horizontal symmetry? If so, is this symmetry consistent with the pattern of quark masses and mixing?

Are the results of neutrino masses and lepton mixing consistent with the quark-lepton symmetry and Grand Unification?

If new light sterile neutrinos exist what is their nature and why are they light?

What are the implications of the neutrino results for GUT, SUSY, models with extra dimensions, and strings? Vice versa: what can these beyond the SM theories tell us about neutrinos?

\section{Bottom-Up}

One can try the "top-down" approach confronting immediately a proposed model with experimental results. Inversely, to get some hints in answering the above questions, it may be worthwhile to try to move bottom-up.

\subsection{Neutrino mass matrix}

There are several steps in the bottom-up approach.

1). Take the results on $\Delta m_{i j}^{2}, \theta_{i j}, m_{e e}$, etc..

2 ). Reconstruct the neutrino mass matrix in the flavor basis (where the charge lepton mass matrix is diagonal) assuming also that neutrinos are Majorana particles. Notice that the mass matrix unifies information contained in masses and mixing angles and this may provide some more hints toward the underlying theory. 
$3)$. Identify the symmetry basis (which may differ from the flavor basis) and the symmetry scale. Take into account the renormalization group effects.

4). Identify the symmetry (as well as mechanism of symmetry violation, if needed) and underlying dynamics.

Let us make the first step in the bottom-up approach. The mass matrix in the flavor basis can be written as

$$
m=U^{*} m^{\operatorname{diag}} U^{+},
$$

where $U=U\left(\theta_{i j}, \delta\right)$ is the mixing matrix, $\delta$ is the Dirac CP-violating phase, and

$$
m^{\operatorname{diag}}=\operatorname{diag}\left(m_{1} e^{-2 i \rho}, m_{2}, m_{3} e^{-2 i \sigma}\right) .
$$

Here $\rho$ and $\sigma$ are the Majorana phases. The mass eigenvalues equal $m_{2}=\sqrt{m_{1}^{2}+\Delta m_{12}^{2}}$, and $m_{3}=$ $\sqrt{m_{1}^{2}+\Delta m_{13}^{2}}$.

The results of reconstruction of the mass matrix $^{49,50}$ are shown in Figs. 9, 10, and 11 as the $\rho-\sigma$ plots for the absolute values of the 6 independent matrix elements. They correspond to three extreme cases: normal mass hierarchy, quasidegenerate spectrum and inverted mass hierarchy. The figures illustrate a variety of possible structures. In particular, for the normal mass hierarchy (Fig. 9) there is clear structure with the dominant $\mu-\tau$ block. Interesting parameterizations of the mass matrix (up to an overall mass factor) are

$$
\left(\begin{array}{lll}
0 & 0 & \lambda \\
0 & 1 & 1 \\
\lambda & 1 & 1
\end{array}\right), \quad\left(\begin{array}{ccc}
\lambda^{2} & \lambda & \lambda \\
\lambda & 1 & 1 \\
\lambda & 1 & 1
\end{array}\right),
$$

where $\lambda \sim 0.2$. Also the matrix similar to the first one in Eq. (14) with $m_{12} \sim \lambda$ and $m_{13} \approx 0$ is possible.

In the case of a quasi-degenerate spectrum, the interesting dominant structures are

$$
\left(\begin{array}{lll}
1 & 0 & 0 \\
0 & 1 & 0 \\
0 & 0 & 1
\end{array}\right), \quad\left(\begin{array}{lll}
1 & 0 & 0 \\
0 & 0 & 1 \\
0 & 1 & 0
\end{array}\right) .
$$

These matrices are realized for values of phases in the corners of the plots: $\rho, \sigma=0, \pi$ (the first matrix) or at $\rho=0, \pi, \sigma=\pi / 2$ (the second one) which corresponds to definite $\mathrm{CP}$-parities of the mass eigenstates. Also the "democratic" structure with equal moduli of elements is possible for the non-trivial values of phases. ${ }^{51}$ Changing the phases one can get any
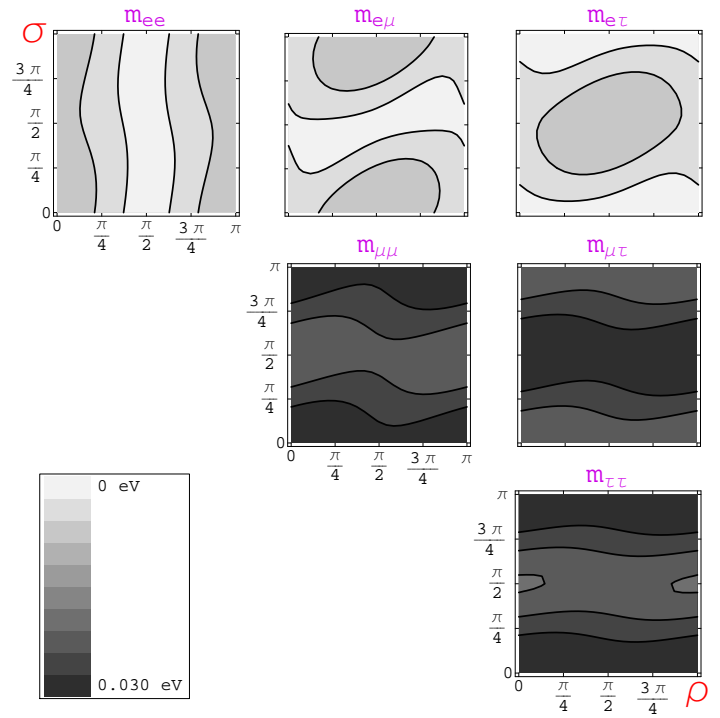

Figure 9. The Majorana mass matrix for the normal mass hierarchy: $m_{3} / m_{2}=5, m_{1} \approx 0.006 \mathrm{eV}$. We show contours of constant mass in the $\rho-\sigma$ plots for the moduli of mass matrix elements. We take for other parameters $\Delta m_{12}^{2}=7 \times 10^{-5} \mathrm{eV}^{2}$, $\Delta m_{13}^{2}=2.5 \times 10^{-3} \mathrm{eV}^{2}, \tan ^{2} \theta_{12}=0.42, \tan \theta_{23}=1, \sin \theta_{13}=$ 0.1 , and $\delta=0$.
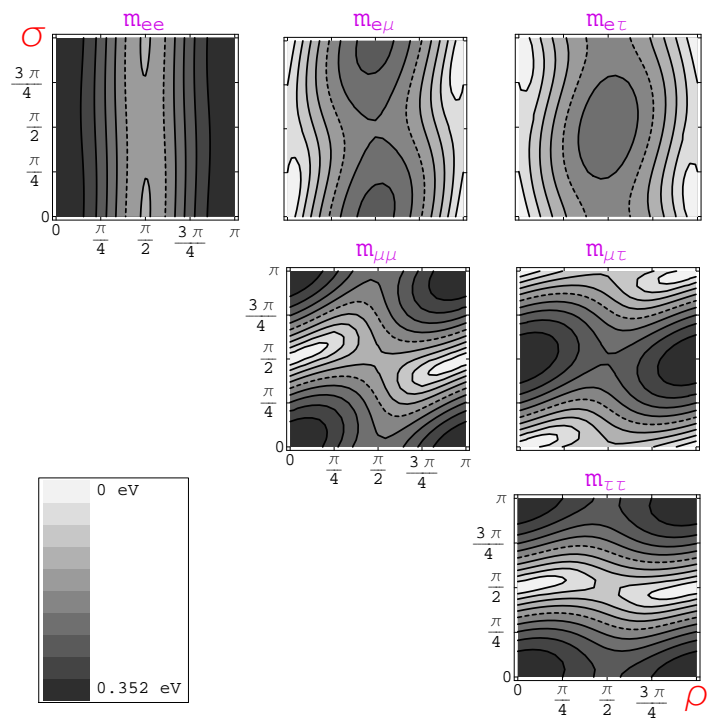

Figure 10. The same as in Fig. 9 for the quasi-degenerate spectrum: $m_{3} / m_{2}=1.01, m_{1} \approx 0.35 \mathrm{eV}$.

intermediate structure between those in Eqs. (14) and (15).

In the case of the inverted hierarchy, generically 


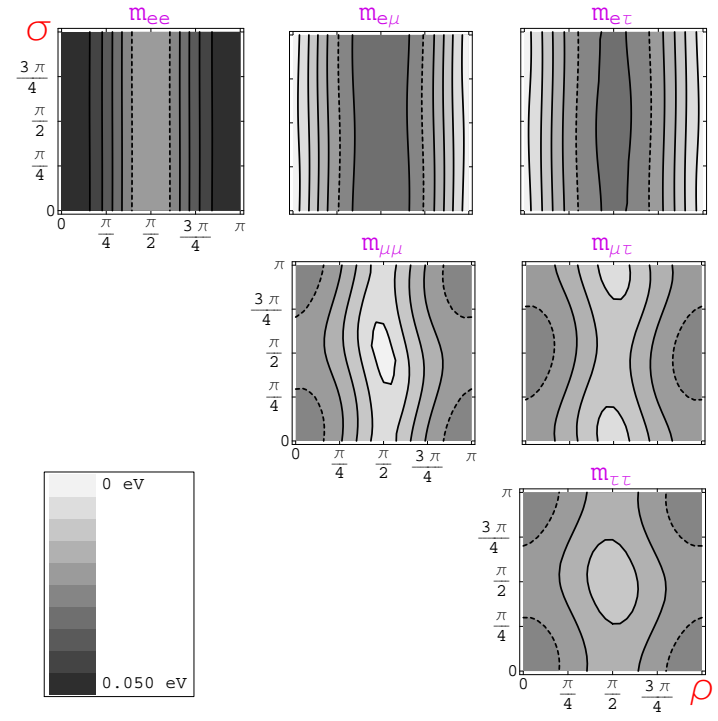

Figure 11. The same as in Fig. 9 for the inverted mass hierarchy: $m_{3} / m_{2}=0.1, m_{3} \approx 0.005 \mathrm{eV}$.

the ee- element is not small. Among interesting examples are

$$
\left(\begin{array}{lll}
0.7 & 1 & 1 \\
1 & 0.1 & 0.1 \\
1 & 0.1 & 0.1
\end{array}\right), \quad\left(\begin{array}{lll}
1 & 0.1 & 0.1 \\
0.1 & 0.5 & 0.5 \\
0.1 & 0.5 & 0.5
\end{array}\right) .
$$

In the SM and MSSM the renormalization group effects do not change the structure of the mass matrix: the corrections to a given element are proportional to the element itself. Furthermore, the corrections are small even in the SUSY case (below $0.1 \%)$. So, unless some new interactions exist, the mass matrix determined at low energies does not change structure when running up to the scale where the corresponding mass operators are formed or up to the symmetry scale.

In contrast to the matrix structure, the radiative corrections are important for the oscillation observables in the case of partially or quasi degenerate mass spectra.

Scanning the $\rho-\sigma$ plots shown in Figs. 9, 10, and 11 , one can make the following observations.

1). A large variety of different structures is still possible, depending strongly on the unknown $m_{1}$, type of mass hierarchy and Majorana phases. The dependence on $\sin \theta_{13}$ and $\delta$ is weak.

2 ). Generically the hierarchy of elements is not strong: within 1 order of magnitude. At the same time matrices with one or two exact zeros are not excluded $^{52}$

3). Matrices are possible with:

- dominant (i) diagonal elements $(\sim I)$, (ii) $\mu \tau$ block, (iii) $e$-row elements, (iv) $e e-, \mu \tau-, \tau \mu-$ elements (triangle structure),

- democratic structure,

- flavor alignment,

- non-hierarchical structures with all elements of the same order,

- flavor disordering,

- zeros at different places, and

- equalities of various element.

4). Typically, the hierarchical structures appear for the Majorana phases near $0, \pi / 2$, or $\pi$.

$5)$. Matrices can be parameterized in terms of powers of small parameter $\lambda=0.2-0.3$ consistent with the Cabibbo mixing.

In a significant part of the parameter space the matrix does not show any regularities, and relative values of its elements appear as random numbers which spread within one order of magnitude. This supports the idea of "Anarchy". ${ }^{53,54}$ Consideration of the anarchy of elements is a test of the possible complexity of the neutrino mass matrix. The case of anarchy can be imitated if neutrino masses have several different contributions, and even if each of them has well defined structure or symmetry, the sum may show up as a matrix with disorder. In this connection one can consider representations of the mass matrix as the sum of matrices, given in Eq. (15), as well as the democratic matrix with certain coefficients. ${ }^{55}$

What is more fundamental: oscillation observables or neutrino mass matrix in some basis? The answer may depend on the type of mass spectrum. In the case of hierarchical spectrum the observables are visibly imprinted into the structure of the mass matrix. In contrast, for the quasi-degenerate spectrum they are just very small perturbations of the dominant structure which is determined by the nonoscillatory parameters: the absolute mass scale and the Majorana CP-violating phases. Then the oscillation parameters can be a result of interplay of some small, in particular, radiative corrections.

In the case of Majorana neutrinos, the elements of the mass matrix are physical parameters: they can be immediately measured in the neutrinoless beta decay and, in principle, in other similar processes. In 
practice, it is not possible to reconstruct the mass matrix from experiment completely. Even in the most optimistic case the phase $\sigma$ will be undetermined and Figs. 9, 10, and 11, give an idea of the remaining uncertainty. Only in the case of the $\mathrm{g}$ mass hierarchy does the dependence on $\sigma$ disappear. The hope is that even without complete reconstruction of the mass matrix we will be able to uncover the underlying physics.

\subsection{Neutrino Mass and Horizontal Symmetry}

Do the results on neutrino masses and mixing indicate certain regularities or symmetry? Can the dominant structures of the mass matrix be explained by a symmetry with the sub-dominant elements appearing as a result of violations of the symmetry? Is the neutrino mass matrix consistent with symmetries suggested for quarks?

The following symmetries have been considered.

1). $L_{e}-L_{\mu}-L_{\tau} \cdot{ }^{56}$ This symmetry supports, in particular, the structure with an inverted mass hierarchy. However, the rather large element $m_{e e}$ (Fig. 11) shows strong violation of this symmetry.

2). Discrete symmetries: $A_{4},{ }^{57} S_{3},{ }^{58} Z_{4}{ }^{59}$, and $D_{4} \cdot{ }^{60}$ They reproduce successfully the dominant structures in Eq. (15) as well as the "democratic" matrix.

However, both classes of symmetries 1) and 2) typically treat quarks and leptons differently.

3). $U(1) \cdot{ }^{61}$ In the Froggatt-Nielsen context ${ }^{62}$ this symmetry can describe mass matrices of both quarks and leptons. However, the claimed predictability of this approach can be questioned: the $U(1)$ charges should be considered as discrete free parameters. Furthermore, precise description of data usually requires coefficients (prefactors) of the order $1(1 / 2-2)$ in front of powers of the expansion parameter. The outcome is that the mixing pattern depends substantially on values of these unknown prefactors.

4). $S U(2),{ }^{63} S O(3),{ }^{64}$, and $S U(3)^{65}$ require a complicated Higgs sector to break the symmetry. Often models are too restrictive and predictions are on the borders of allowed regions.

The question is still open. Different symmetries are consistent with the neutrino data. But realizations of these symmetries in specific models are not simple. The hope is that future neutrino data (better knowledge of the mass matrix) can discriminate among possibilities.

\section{How We May Go...}

\subsection{Neutrality and Mass}

In answering the questions of Sec. 3 one can implement the "minimalistic" approach, that is, to try to relate features of the neutrino masses and mixings with already known differences of characteristics of neutrinos and other fermions.

The main feature of neutrinos is neutrality:

$$
Q_{\gamma}=Q_{c}=0 .
$$

It leads to the following possibilities:

- neutrinos can be Majorana particles;

- they can mix with singlets of the SM symmetry group; and

- the right-handed components $(\mathrm{RH})$, if they exist, are singlets of $S U(3) \times S U(2) \times U(1)$. So, their masses are unprotected by the symmetry and therefore can be large.

In turn, properties of the $\mathrm{RH}$ components open two other possibilities. The RH neutrinos can:

- have large Majorana masses: $M_{R} \gg V_{E W}$ (which leads to the see-saw); and

- propagate in (large, or warped, or infinite) extra dimensions, or be located on the "hidden" (not ours) brane in contrast to other fermions.

Introduction of the $\mathrm{RH}$ neutrino has a number of attractive features, ${ }^{66}$ in particular, it allows one to extend the electroweak symmetry to the gauged $S U(2)_{L} \times S U(2)_{R} \times U(1)_{B-L}$.

Is this enough to explain the properties of the mass spectrum and mixings?

\subsection{Effective Operator}

Suppose the SM particles are the only light degrees of freedom. Then at low energies (after integrating out the heavy degrees of freedom) one can get the operator: ${ }^{67}$

$$
\frac{\lambda_{i j}}{M}\left(L_{i} H\right)^{T}\left(L_{j} H\right), \quad i, j=e, \mu, \tau,
$$


where $L_{i}$ is the lepton doublet, $\lambda_{i j}$ are the dimensionless couplings and $M$ is the cut-off scale. After EW symmetry breaking it generates the neutrino masses

$$
m_{i j}=\frac{\lambda_{i j}\langle H\rangle^{2}}{M} .
$$

For $\lambda_{i j} \sim 1$ and $M=M_{P l}$ we find $m_{i j} \sim$ $10^{-5} \mathrm{eV}^{68}$ Three important conclusions immediately follow from this consideration.

1). The Planck scale (gravitational) interactions are not enough to generate the observed values of the masses. So, new scales of physics below $M_{P l}$ should exist.

2). Contributions to the neutrino masses of the order $\sim 10^{-5} \mathrm{eV}$ are still relevant for phenomenology. Sub-dominant structures of the mass matrix can be generated by the Planck scale interactions. ${ }^{69}$

3 ). The neutrino mass matrix can get observable contributions from all possible energy/mass scales from the EW scale to the Planck scale. As a consequence, the structure of the mass matrix can be rather complicated.

\subsection{See-saw}

The see-saw (type I) mechanism ${ }^{70}$ implements the neutrality in full strength (Majorana nature, heavy $\mathrm{RH}$ components). Introducing the Dirac mass matrix, $m_{D}=Y v_{E W}$, where $Y$ is the matrix of Yukawa couplings and $v_{E M}$ is the electroweak VEV, we have

$$
\left.m=-m_{D}^{T} M_{R}^{-1} m_{D} \quad \text { (type } I\right) .
$$

If the $S U(2)$ triplet, $\Delta_{L}$, exists which develops a $\operatorname{VEV}\left\langle\Delta_{L}\right\rangle$, the left-handed neutrinos can get a direct mass $m_{L}$ via the interaction $f_{\Delta} L^{T} L \Delta_{L}$. If $\Delta_{L}$ is very heavy, it can develop the induced VEV from interactions with a doublet: $\left\langle\Delta_{L}\right\rangle=v_{E W}^{2} / M$. So that

$$
m_{L}=f_{\Delta} \frac{v_{E W}^{2}}{M} \quad(\text { typeII }),
$$

and here we deal with the see-saw of VEV's. ${ }^{71}$

In $S O(10)$ with $126_{H}$-plet of Higgses we have $M_{R}=f v_{R}$, where $f$ is the Yukawa coupling of the matter 16-plet with $126_{H}$ and $v_{R}$ is the VEV of the $S U(5)$ singlet component of $126_{H}$. Now $f_{\Delta}=f$, and the general mass term which contains both types of contributions can be written as

$$
m=\frac{v_{E M}^{2}}{v_{R}}\left(f \lambda-Y^{T} f^{-1} Y\right) .
$$

Here $\lambda$ is the coupling of 10 - and 126-plets. According to this expression the flavor structure of the two contributions may partially correlate.

The number of RH neutrinos can differ from 3 . Two possibilities have been explored:

"... less than 3": which corresponds to the $3 \times 2$ see-saw in the case of two RH neutrinos. ${ }^{72}$ Such a possibility can be realized in the limit when one of the RH neutrinos is very heavy: $M \sim M_{P l}$, being, e.g. unprotected by the $S U(2)_{H}$ horizontal symmetry. It leads to one exactly massless LH neutrino and smaller number of free parameters.

One can further reduce the number of unknown parameters postulating zeros in the Dirac matrix $m_{D} .{ }^{52}$ This can lead to the predictions for $\sin \theta_{13}$, $m_{e e}$, as well as for relations between $\delta$ and the phase responsible for leptogenesis.

"... more than 3 ": additional singlets of the SM may not be related to the family structure. Alternatively, three additional singlets, $S$, which belong to families, can couple to the RH neutrinos. In the latter case the double see-saw can be realized. ${ }^{73}$ In the basis $\left(\nu, \nu^{c}, S\right)$, the mass matrix may have the form

$$
\left(\begin{array}{lll}
0 & m_{D} & 0 \\
m_{D}^{T} & 0 & \mathrm{M} \\
0 & M^{T} & \mu
\end{array}\right)
$$

which leads to the light neutrino masses:

$$
m=-m_{D}^{T}\left(M^{-1}\right)^{T} \mu M^{-1} m_{D} .
$$

Two interesting limits are: (i) $\mu \ll M$, it allows one to reduce all high mass scales for the same values of the light neutrino masses, (ii) $\mu \gg M$, e.g. $\mu=M_{P l}$, and $M=M_{G U}$ : in this case the intermediate mass scale, $M_{G U}^{2} / M_{P l}=10^{12}-10^{14} \mathrm{GeV}$ for the masses of $\mathrm{RH}$ neutrinos can be obtained.

\subsection{Grand Unification and Neutrino Mixing}

GU theories provide a large mass scale comparable to the scale of RH neutrino masses. ${ }^{74}$ Furthermore, one can argue that GUT + see-saw can naturally lead to the large lepton mixing in contrast to the quark mixing. The arguments go like this:

1. Suppose that all quarks and leptons of a given family are in a single multiplet $F_{i}$ (as 16 of $\mathrm{SO}(10)$ ).

2. Suppose that all Yukawa couplings are of the same order thus producing matrices with generically large mixing. 
3. If the Dirac masses are generated by an unique Higgs multiplet, say $10_{H}$ of $\mathrm{SO}(10)$, the mass matrices of the up and down components of the weak doublets have identical structures, and so, will be diagonalized by the same rotations. As a result:

- no mixing appears for quarks, and

- masses of up and down components will be equal to each other (this needs to be corrected).

4. In contrast to other fermions, the $\mathrm{RH}$ neutrinos acquire Majorana masses via the additional Yukawa couplings (with $126_{H}$ of $\mathrm{SO}(10)$ ).

5. If those (Majorana type) Yukawa couplings are also of the generic form, they produce $M_{R}$ with large mixing which leads then to large lepton mixing.

The problem of this scenario is the strong hierarchy of the quark and lepton masses. Indeed, taking the neutrino Dirac masses as $m_{D}=$ $\operatorname{diag}\left(m_{u}, m_{c}, m_{t}\right)$ in a spirit of GU, we find that for generic $M_{R}$ the see-saw type I formula (20) produces strongly hierarchical mass matrix with small mixings. Possible solutions are:

1. a special structure of $M_{R}$ which compensates the strong hierarchy in $m_{D}$;

2. a substantial difference in the Dirac matrices of quarks and leptons: $m_{D}(q) \neq m_{D}(l)$; or

3. a type II see-saw for which there is no relation to $m_{D}$.

In what follows we will comment on these three possibilities.

See-Saw enhancement of mixing. ${ }^{75}$ Can the same mechanism (see-saw) which explains the smallness of the neutrino mass also explain the large lepton mixing? So, that the large mixing appears as an artifact of the see-saw?

The idea is that due to the (approximate) quarklepton symmetry, the Dirac mass matrices of the quarks and leptons have the same (similar) structure $m_{D} \sim m_{\text {up }}, m_{l} \sim m_{\text {down }}$ leading to small mixing in the Dirac sector. However, the special structure of $M_{R}$ (which has no analogue in the quark sector) leads to an enhancement of lepton mixing. Two different possibilities have been found:

- strong (nearly quadratic) hierarchy of the $\mathrm{RH}$ neutrino masses: $M_{i R} \sim\left(m_{\text {iup }}\right)^{2}$; and
- strong interfamily connection (pseudo Dirac structures) like

$$
M_{R}=\left(\begin{array}{lll}
\mathrm{A} & 0 & 0 \\
0 & 0 & \mathrm{~B} \\
0 & \mathrm{~B} & 0
\end{array}\right) .
$$

In the three neutrino context both possibilities can be realized simultaneously, so that the pseudo Dirac structure leads to maximal 2-3 mixing, whereas the strong hierarchy $A \ll B$ enhances the 1-2 mixing.
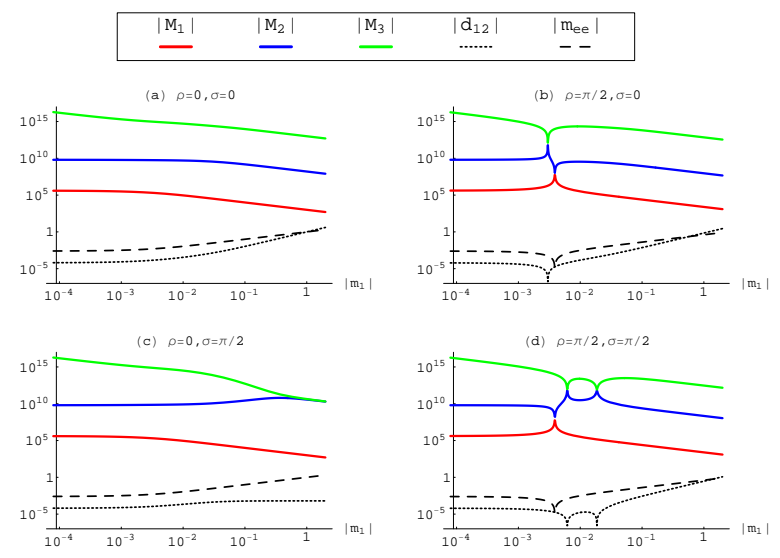

Figure 12. The masses of the $\mathrm{RH}$ neutrinos in $\mathrm{GeV}$ as functions of the lightest neutrino mass $\left|m_{1}\right|$ in $\mathrm{eV}$ (solid lines) for different values of the Majorana phases of light neutrinos. ${ }^{75}$ We take $\sin \theta_{13}=0$ and the best-fit values of other oscillation parameters. Shown is also the dependence of $m_{e e}$ (in $\mathrm{eV}$ ) on $\left|m_{1}\right|$ (thin dashed line).

In Fig. 12 we show dependences of the RH neutrino masses reconstructed from the low energy data on the lightest neutrino mass for different values of the Majorana phases. According to this figure

$1)$. In the largest part of the parameter space $\left(m_{1}, \rho, \sigma\right)$ there is a very strong $(4-5$ orders of magnitude) mass hierarchy of the $\mathrm{RH}$ neutrinos.

2). The lightest mass is typically below $10^{5} \mathrm{GeV}$, thus strongly violating the lower bound on the mass from the condition of successful leptogenesis: $M_{1}>$ $4 \times 10^{8} \mathrm{GeV}{ }^{77}$

3). At certain points the level crossings occur. At these points (i) there is a strong degeneracy of mass eigenstates: in particular, $M_{1}=M_{2}$, (ii) the lightest mass can be as large as $10^{8} \mathrm{GeV}$ and (iii) the lepton asymmetry can be resonantly enhanced ${ }^{78}$ up to the required value.

Large mixing and type II see-saw. In general, the structure of neutrino mass matrix generated by the 
type II (triplet) see-saw is not related to structures of matrices of other fermions.

In some particular cases, however, the relations can appear leading to interesting consequences. In the $\mathrm{SO}(10)$ model the $126_{H}$ Higgs multiplet can play a double role: (i) generate neutrino masses $m_{L}=Y_{126} v_{\Delta}$, where $v_{\Delta}$ is the $\mathrm{VEV}$ of the $\mathrm{SU}(2)$ triplet in $126_{H}$; and (ii) give contributions to the quark and charged lepton masses (if doublets contained in 126 get VEV's) reproducing the GeorgiJarlskog mass relation for the first and second generations.

Since $m_{b}-m_{\tau} \propto\left(Y_{126}\right)_{33}$, the contribution of $126_{H}$ destroys the $b-\tau$ unification unless $\left(Y_{126}\right)_{33} \leq$ $\left(Y_{126}\right)_{23}$. The latter leads to large (but not necessarily maximal) $2-3$ lepton mixing. In this context, the $b-\tau$ unification implies large 2-3 mixing. ${ }^{79}$

In models of this type a successful leptogenesis is possible with participation of the scalar triplet. ${ }^{80}$ The model has been generalized also to 3 generations, leading typically to 1-2 mixing at the larger side of the allowed region. ${ }^{81}$

Single RH neutrino dominance. ${ }^{82}$ The large neutrino mixing and relatively strong mass hierarchy implied by the solar and atmospheric neutrino data can be reconciled if only one $\mathrm{RH}$ neutrino gives the dominant contribution to the see-saw. (This leads to the submatrix of $m_{L}$ with nearly zero determinant.) There are two different realizations of this possibility. In one case the large mixing originates from the large mixing in the Dirac neutrino mass matrix $m_{D}$ : two LH neutrinos have nearly equal couplings to the dominating $\mathrm{RH}$ component. Suppose that $\left(m_{D}\right)_{23} \approx\left(m_{D}\right)_{33}=m,\left(m_{D}\right)_{13}=\lambda m$ $(\lambda \approx 0.2)$ and all other elements of $m_{D}$ are much smaller. Then if only $\left(M^{-1}\right)_{33}$ is large in the inverted matrix, the see-saw leads to the mass matrix which reproduces the second structure in Eq. (14).

In another version, the dominance is realized when two RH neutrinos are much heavier than the third (dominating) one and no large mixing in $m_{D}$ appears. This is equivalent to the strong mass hierarchy case of the see-saw enhancement mechanism. A realization requires $\left(m_{D}\right)_{22} \approx\left(m_{D}\right)_{23} \ll\left(m_{D}\right)_{33}$, and $\left(M^{-1}\right)_{22}$ being the dominant element.

Lopsided models. ${ }^{83}$ Large lepton mixing follows from the charge lepton mass matrix which should be non-symmetric (no left-right symmetry). This does not contradict the Grand Unification: in $\mathrm{SU}(5)$ the
LH components of leptons are unified with the $\mathrm{RH}$ components of quarks: $5=\left(d^{c}, d^{c}, d^{c}, l, \nu\right)$. Therefore large mixing of the $\mathrm{LH}$ leptonic components is accompanied by large mixing of the $\mathrm{RH} d$-quarks which is unobservable. Introducing the Dirac mass matrix of the charged leptons with the only large elements $\left(m_{l}\right)_{33} \sim\left(m_{l}\right)_{23}$, one can obtain the large 2-3 lepton mixing. This scenario can also be realized in $S O(10)$, if the symmetry is broken via $S U(5)$. A double lopsided matrix for both large mixing is also possible.

Radiative enhancement of mixing. ${ }^{84}$ The idea is that the difference between the quark and lepton mixings is a result of different renormalization group effects. The lepton mixing is small (similar to quark mixing) at the GU scale but running to low energies leads to its enhancement.

The main requirement of such an enhancement is that the neutrino mass spectrum is quasi-degenerate (and this is the key point which distinguishes quarks and leptons). The enhancement occurs when neutrinos become even more degenerate at low energies. For instance, running of the $2-3$ mixing is described by

$$
\frac{d \sin \theta_{23}}{d t} \sim\left(\sin \theta_{12} U_{\tau 1} D_{31}-\cos \theta_{12} U_{\tau 2} D_{32}\right)
$$

where $t \equiv 1 / 8 \pi^{2} \log (q / M), D_{i j} \equiv\left(m_{i}+m_{j}\right) /\left(m_{i}-\right.$ $\left.m_{j}\right)$, and $m_{i}$ are the mass eigenvalues. The minus sign in the denominators of $D_{i j}$ plays the key role.

The mechanism requires fine tuning of the initial mass splittings and radiative corrections. In principle, in the MSSM both the 1-2 and 2-3 mixings can be enhanced in this way. In the SM only 1-2 mixing can be enhanced.

The fine-tuning problem can be avoided if the masses are generated from the Kähler potential. In this case large mixing appears as the infrared fixed point. $^{85}$

Another possible application of the radiative effects is the generation of small oscillation parameters like $\Delta m_{12}^{2}$, and $\sin \theta_{13} \cdot{ }^{86}$ Again this can be realized only in the case of a mass spectrum with degeneracy.

\subsection{How To Test the See-Saw Mechanism?}

This is the key question which implies essentially the test of existence of the heavy Majorana RH neutrinos. There are two (known) possibilities. 
1). Leptogenesis ${ }^{87}$ For the hierarchical RH neutrino spectrum and in assumption of the type I seesaw, it gives bounds on (i) the mass of the lightest $\mathrm{RH}$ neutrino $M_{R 1}$, (ii) the effective parameter $\tilde{m}_{1}$ which determines the washout effect. Notice that the leptogenesis probes the combination of the Yukawa couplings $\left(Y Y^{\dagger}\right)_{i i}$.

$2)$. The RH neutrinos can produce renormalization effects above the scale of their masses: between $M_{R}$ and, say, the GUT scale. In particular, they can renormalize the $m_{b}-m_{\tau}$ mass relation ${ }^{88}$ which leads to the observable effect in the assumption of $m_{b}-m_{\tau}$ unification at the GUT scale.

Another possibility is that the renormalization due to RH neutrinos modifies masses and mixing of the light neutrinos, e.g. enhances the lepton mixing. ${ }^{89}$

\subsection{SUSY See-Saw}

Additional possibilities to test the see-saw mechanism appear if SUSY is realized. The part of superpotential relevant for the see-saw can be written as

$$
W_{l e p}=l^{c T} Y_{l} L H_{1}+\nu^{c T} Y L H_{2}+\frac{1}{2} \nu^{c T} M_{R} \nu^{c} .
$$

Structures relevant for the see-saw are imprinted into properties of the slepton sector. So, studying the properties of sleptons (masses, decay rates, etc.) one can get information about the neutrino mass generation.

Certain predictions can be made in the assumptions of universal soft SUSY breaking masses $\left(m_{0}\right.$, $A_{0}$ ) at high (GUT ?) scale $M_{X}$, and the absence of new particles/interactions up to $M_{X}$.

Due to renormalization group effects the Yukawa couplings (27) give contributions to the masses of left sleptons at low energies: ${ }^{90}$

$\left(m_{S}^{2}\right)_{a b}=m_{a}^{2} \delta_{a b}-\frac{3 m_{0}^{2}+A_{0}^{2}}{8 \pi^{2}}\left(Y^{\dagger}\right)_{a i}(Y)_{i b} \log \left(\frac{M_{X}}{M_{i R}}\right)$.

The contribution splits masses of sleptons of different flavors and sleptons-antisleptons as well as leading to mixing of sleptons (the off-diagonal terms in Eq. (28)) which is related to the mixing of neutrinos.

In turn, these contributions to the slepton masses produce a number of observable effects:

1. rare leptonic decays: the one-loop mixing of sleptons of different flavors induces the flavor violating decays: $\mu \rightarrow e \gamma, \tau \rightarrow \mu \gamma, \tau \rightarrow e \gamma{ }^{90}$

2. sneutrino flavor oscillations; ${ }^{91}$

3. slepton decays; ${ }^{92}$

4. sneutrino-antisneutrino oscillations; ${ }^{93}$ and

5. contribution to the electric dipole moments of charged leptons. ${ }^{94}$

Up to a log factor these effects are determined by the combination $\sim\left(Y^{\dagger} Y\right)$. Notice that another combination: $\left(Y^{T} M^{-1} Y\right)$ enters the see-saw (type I) formula. It was shown ${ }^{95}$ that knowledge of these combinations allows, in principle, one to reconstruct parameters of the $\mathrm{RH}$ neutrino sector (masses, phases). For this, in turn, one needs to reconstruct completely the mass matrix of light neutrinos, discover SUSY and measure rare processes with high enough accuracy. This looks practically impossible, at least now.

Partial tests of the see-saw can be done by studying the rare decays induced by the slepton mixing. The branching ratio equals

$$
B(\mu \rightarrow e \gamma)=\frac{\alpha^{3}}{G_{F}^{2} m_{S U S Y}^{8}}\left|\left(m_{S}^{2}\right)_{\mu e}\right|^{2} \tan ^{2} \beta,
$$

where $m_{S U S Y}=m_{S U S Y}\left(m_{0}, m_{1 / 2}\right)$ is the effective SUSY mass parameter, $m_{1 / 2}$ is the gaugino mass, $\tan \beta$ is the ratio of MSSM Higgs doublet VEV's, and $\left(m_{S}^{2}\right)_{\mu e}$ is given in Eq. (28).

If the large lepton mixing originates from the Dirac mass matrix (lopsided models, versions of the single RH neutrino dominance), the Yukawa couplings $Y_{\mu i}, Y_{e i}$ are large and for $m_{S U S Y} \sim 200$ $\mathrm{GeV}$ the branching ratio in Eq. (29) turns out to be $10^{-12}-10^{-11}$ - at the level of the present experimental bound. Determination of $m_{S U S Y}$ in terms of $m_{0}$ and $m_{1 / 2}$ beyond the leading log approximation has further enhanced the branching ratio. ${ }^{96}$

\subsection{Other Mechanisms}

What are other possibilities apart from the see-saw? The incomplete list includes.

1. Various radiative mechanisms: The Zee (one loop) mechanism ${ }^{97}$ is essentially excluded in its minimal version by data. ${ }^{98}$ One loop generation also occurs in the SUSY models with trilinear R-parity violating couplings. Neutrino masses can be generated in two loops as suggested by Zee ${ }^{97}$ and Babu. ${ }^{99}$

2. Neutrino mass generation by the bi-linear Rparity violation terms. ${ }^{100}$ This mechanism is a combination of the see-saw and radiative effects: neutrino 
mass appears as the see-saw due to mixing of neutrinos with neutralinos (Higgsino) and the latter is generated by running from the high mass scales.

3. Mechanisms related to the existence of extra dimensions. There are different scenarios: (i) large extra dimensions (ADD) $)^{101}$ where the Dirac neutrino mass is suppressed by the large volume of extra dimensions, (ii) warped extra dimensions (RS), where the RH neutrinos can be zero modes localized on the hidden brane, thus leading again to the small Dirac neutrino mass, ${ }^{102}$ and (iii) infinite extra dimensions. ${ }^{103}$

4). There are several new proposals which implement various realizations of the see-saw mechanism.

Models with dynamical symmetry breaking 104 reproduce the low scale see-saw mechanism with the RH neutrino masses below the symmetry breaking scale, and correspondingly, with small Dirac masses (much smaller than the masses of quarks and charged leptons).

Also in models with "Little Higgs" 105 the neutrinos get masses via the low scale see-saw.

In models with dimensional deconstruction ${ }^{106}$ the see-saw scale (masses of the RH neutrinos) is determined by the inverse lattice spacing. The Dirac mass matrix is nearly diagonal and the large (maximal) mixing follows from the pseudo-Dirac structures in the RH mass matrix which correspond to $L_{e}-L_{\mu}-L_{\tau}$ symmetry. Essentially, the maximal mixing appears because different lepton families belong to different sites of the lattice and the link scalar fields (singlets of SM) couple with the $\mathrm{RH}$ components of neutrinos, thus producing the off-diagonal (in flavor space) Majorana mass terms.

These alternative mechanisms imply deviation from minimality. They can accommodate the neutrino masses and produce some interesting features. However, they do not really lead to a better understanding of the experimental results and require introduction of additional elements (physics) beyond the Standard Model with RH neutrinos.

Turning the arguments: the neutrino data can be used to put limits on the suggested alternative mechanisms, and consequently, on physics beyond the SM. In such a way the neutrinos can probe extra dimensions, dynamical symmetry breaking, etc..

\section{Conclusions}

During last several years enormous progress has been achieved in the determination of the neutrino masses and mixings and in studies of the neutrino mass matrix. Still, large freedom exists in the possible structures of the mass matrix which leads to very different interpretations of the results. There are no definite hints from the bottom-up approach yet, and more information is needed, in particular, on the type of mass spectrum.

The main question (still open) is: what is behind the obtained results? What is the underlying physics? Preference? Probably, the see-saw associated to the Grand Unification. The context of $S O(10)$ looks rather appealing in spite of known problems. Other mechanisms (being in a less advanced stage of development) are not excluded and can give leading or sub-leading contributions to neutrino mass.

How can ideas about neutrinos be checked? Future experiments will perform precision measurements of neutrino parameters. Apart from this to understand the underlying physics we will certainly need results from the non-neutrino experiments:

- astrophysics and cosmology;

- searches for rare processes like flavor violating lepton decays, proton decay, etc.; and

- future high energy colliders.

\section{Acknowledgments}

I am grateful to E. Kh. Akhmedov, P. de Holanda, M. Frigerio, C. Lunardini and O. Peres for fruitful discussions and help in preparation of this talk.

\section{References}

1. SNO collaboration (Q. R. Ahmad et al.), nuclex/0309004.

2. J. N. Bahcall, M.H. Pinsonneault and S. Basu, Astrophys. J. 555, 990 (2001).

3. L. Wolfenstein, Phys. Rev. D 17, 2369 (1978); in "Neutrino-78", Purdue Univ., C3, (1978). S. P. Mikheyev and A. Yu. Smirnov, Yad. Fiz. 42, 1441 (1985); Nuovo Cim. C 9, 17 (1986); Sov. Phys. JETP, 64, 4 (1986).

4. A. B. Balantekin and H. Yüksel, hep-ph/0309079.

5. G.L. Fogli, E. Lisi, A. Marrone, A. Palazzo, hep$\mathrm{ph} / 0309100$. 
6. M. Maltoni, T. Schwetz, M. A. Tortola, J.W.F. Valle, hep-ph/0309130 (v.2).

7. P. Aliani, V. Antonelli, M. Picariello, E. TorrenteLujan, hep-ph/0309156.

8. P. Creminelli, G. Signorelli, A. Strumia, hepph/0102234, v.5, Sept. 15 (2003).

9. A. Bandyopadhyay, S. Choubey, S. Goswami, S. T. Petcov, D.P. Roy, hep-ph/0309174.

10. P. C. de Holanda, A.Yu. Smirnov, hep-ph/0309299.

11. K. Eguchi et al., (KamLAND), Phys. Rev. Lett. 90, 021802 (2003).

12. CHOOZ Collaboration, M. Apollonio et al., Phys. Lett. B 466, 415 (1999); Eur. Phys. J. , C 27, 331 (2003).

13. B. T. Cleveland et al., Astroph. J. 496, 505 (1998).

14. Y. Fukuda et al., (Super-Kamiokande) Phys. Rev. Lett. 86, 5651 (2001), ibidem, 82, 5656 (2001), Phys. Lett. B 539, 179 (2002); hep-ex/0309011.

15. P. C. de Holanda, A.Yu. Smirnov, hep-ph/0307266.

16. BOREXINO Collaboration, G. Alimonti et. al., Astropart. Phys. 16, 073022 (2002).

17. See talks at Low $4 \mathrm{Nu} 4$ th International Workshop on Low Energy and Solar Neutrinos, Paris, France, May 19-21 (2003), http://cdfpc53.in2p3.fr/LowNu2003/.

18. Super-Kamiokande Collaboration, Y. Hayato, talk given at the HEP2003 International Europhysics Conference (Aachen, Germany, 2003), website: eps2003.physik.rwth-aachen.de .

19. G.L. Fogli, E. Lisi, A. Marrone, D. Montanino, A. Palazzo, A.M. Rotunno, hep-ph/0308055.

20. O. L. G. Peres, A.Yu. Smirnov, Phys. Lett. B 456, 204 (1999); hep-ph/0309312.

21. M. C. Gonzalez-Garcia, C. Pena-Garay, hepph/0306001.

22. Y. Farzan, A.Yu. Smirnov, Phys. Rev. D 65, 113001 (2002).

23. K. Hirata et al., Phys. Rev. Lett. 58, 1490 (1987); R. M. Bionta et al., Phys. Rev. Lett. 58, 1494 (1987); E. N. Alekseev, et al., JETP Lett. 45, 589 (1987).

24. A. S. Dighe, A. Yu. Smirnov, Phys. Rev. D 62, 033007 (2000).

25. C. Lunardini, A.Yu. Smirnov, Phys. Rev. D 63, 073009, (2001); M. Kachelriess et al., Phys. Rev. D 65, 073016 (2002).

26. C. Lunardini, A.Yu. Smirnov, (in preparation).

27. A. Yu. Smirnov, D. N. Spergel, J. N. Bahcall, Phys. Rev. D 49, 1389 (1994); H. Minakata, H. Nunokawa, Phys. Lett. B 504, 301 (2001).

28. F. Feruglio, A. Strumia, F. Vissani, Nucl. Phys. B 637, 345 (2002), Addendum-ibid., B 659, 359 (2003).

29. H.V. Klapdor-Kleingrothaus et al., Eur. Phys. J. A 12, 147 (2001), A. M. Bakalyarov et al., talk given at the 4 th International Conference on Non-accelerator New Physics (NANP 03), Dubna, Russia, 23-28 Jun. 2003, hep-ex/0309016.

30. H.V. Klapdor-Kleingrothaus et al., Mod. Phys. Lett. A 16, 2409 (2001).

31. D. N. Spergel et al., Astrophys. J. Suppl., 148, 175
(2003), [astro-ph/0302209].

32. O. Elgaroy, O. Lahav, JCAP 0304, 004 (2003).

33. S. Hannestad, JCAP 0305, 004 (2003).

34. S. W. Allen, R. W. Schmidt and S. L. Bridle, astro$\mathrm{ph} / 0306368$.

35. A. Aguilar et al., (LSND Collaboration) Phys. Rev. D 64, 112007 (2001).

36. B. Armbruster, et al., (KARMEN), Phys. Rev. Lett. 90, 181804 (2003).

37. K. S. Babu and S. Pakwasa, hep-ph/0204226.

38. G. Barenboim, L. Borissov, J. Lykken, hepph/0212116.

39. A. Strumia, Phys. Lett. B 539, 91 (2002).

40. M.C. Gonzalez-Garcia, M. Maltoni T. Schwetz, Phys. Rev. D 68, 053007 (2003).

41. O. L. G. Peres, A.Yu. Smirnov, Nucl. Phys. B 599, 3 (2001).

42. M. Sorel, J. Conrad, M. Shaevitz, hep-ph/0305255.

43. F. Dydak et al., Phys. Lett. B 134, 218 (1984).

44. A. Romosan et al., Phys. Rev. Lett. 78, 2912 (1997).

45. P. Astier et al., [NOMAD Collaboration] Nucl. Phys. B 611, 3 (2001).

46. A. Bazarko, hep-ex/0210020.

47. J. Conrad, private communication.

48. V. Barger, D. Marfatia, K. Whisnant, hepph/0308299.

49. See for review: G. Altarelli, F. Feruglio, Phys. Rept. 320, 295 (1999), Phys. Lett. B 439, 112 (1998).

50. M. Frigerio, A. Yu. Smirnov, Nucl. Phys. B 640, 233 (2002), Phys. Rev. D 67, 013007 (2003).

51. H. Fritzsch and Z. Z. Xing, Phys. Lett. B 372, 265 (1996), ibidem 440, 313 (1998); G.C. Branco, J.I. Silva-Marcos, Phys. Lett. B 526, 104 (2002).

52. P. H. Frampton, S. L. Glashow and D. Marfatia, Phys. Lett. B 536, 79 (2002).

53. L. J. Hall, H. Murayama and N. Weiner, Phys. Rev. Lett. 84, 2572 (2000); N. Haba and H. Murayama, Phys. Rev. D 63, 053010 (2001); A. de Gouvea and H. Murayama, hep-ph/0301050; J. R. Espinosa, hepph/0306019.

54. F. Vissani, Phys. Lett. B 508, 79 (2001); M. Hirsch and S. F. King, Phys. Lett. B 516, 103 (2001); G. Altarelli, F. Feruglio and I. Masina, JHEP 0301, 035 (2003).

55. P.F. Harrison, W.G. Scott, Phys. Lett. B 557, 76 (2003); E. Ma, hep-ph/0308282.

56. S. T. Petcov, Phys. Lett. B 110, 245 (1982), R. Barbieri et al., JHEP 9812, 017 (1998).

57. E. Ma, G. Rajasekaran, Phys. Rev. D 64 113012, (2001); K.S. Babu, E. Ma, J.W.F. Valle, Phys. Lett. B 552, 207 (2003).

58. J. Kubo et al., Prog. Theor. Phys. 109, 795 (2003).

59. E. Ma, G. Rajasekaran, hep-ph/0306264.

60. W. Grimus, L. Lavoura, hep-ph/0305046.

61. J. Bijnens C. Wetterich, Nucl. Phys. B 292, 443 (1987); M. Leurer, Y. Nir and N. Seiberg, Nucl. Phys. B 398, 319 (1993), ibidem, 420, 468 (1994); L. E. Ibanez and G.G. Ross, Phys. Lett. B 332, 100 
(1994); P. Binetruy and P. Ramond, Phys. Lett. B 350, 49 (1995), for references and recent discussion see G. Altarelli and F. Feruglio, hep-ph/0306265.

62. C. D. Froggatt and H. B. Nielsen, Nucl. Phys. B 147, 277 (1979).

63. R. Kuchimanchi and R. N. Mohapatra, Phys. Rev. D 66, 051301 (2002).

64. R. Barbieri, L. J. Hall, G. L. Kane and G. G. Ross, hep-ph/9901228.

65. G. Kribs, hep-ph/0304256; S. F. King, Phys. Lett. B 520, 243 (2001); S.F. King, G.G. Ross, Phys. Lett. B 574, 239 (2003).

66. R. E. Marshak and R. N. Mohapatra, Phys. Lett. B 91, 222 (1980).

67. S. Weinberg, Phys. Rev. Lett. 43, 1566 (1979).

68. R. Barbieri, J. Ellis and M. K. Gaillard, Phys. Lett. B 90249 (1980); E. Kh. Akhmedov, Z. G. Berezhiani, G. Senjanović, Phys. Rev. Lett. 69, 3013 (1992).

69. F. Vissani, M. Narayan, V. Berezinsky, Phys. Lett. B 571, 209 (2003).

70. M. Gell-Mann, P. Ramond and R. Slansky, in Supergravity, eds P. van Niewenhuizen and D. Z. Freedman (North Holland, Amsterdam 1980); P. Ramond, Sanibel talk, retroprinted as hep-ph/9809459; T. Yanagida, in Proc. of Workshop on Unified Theory and Baryon number in the Universe, eds. O. Sawada and A. Sugamoto, KEK, Tsukuba, (1979); S. L. Glashow, in Quarks and Leptons, Cargèse lectures, eds M. Lévy, (Plenum, 1980, New York) p. 707; R. N. Mohapatra and G. Senjanović, Phys. Rev. Lett. 44, 912 (1980).

71. R. N. Mohapatra and G. Senjanović, Phys. Rev. D 23, 165 (1981), C. Wetterich, Nucl. Phys. B 187, 343 (1981).

72. P. H. Frampton, S. L. Glashow, T. Yanagida, Phys. Lett. B 548, 119 (2002).

73. R. N. Mohapatra and J. W. F. Valle, Phys. Rev. D 34, 1642 (1986).

74. For recent review of neutrino masses in GUT see MC. Chen and K. T. Mahanthappa, hep-ph/0305088.

75. A. Yu. Smirnov, Phys. Rev. D 483264 (1993); M. Tanimoto, Phys. Lett. B 345, 477 (1995); T.K. Kuo, Guo-Hong Wu, Sadek W. Mansour, Phys. Rev. D 61, 111301 (2000); G. Altarelli F. Feruglio and I. Masina, Phys. Lett. B 472, 382 (2000); S. Lavignac, I. Masina, C. A. Savoy, Nucl. Phys. B 633, 139 (2002). A. Datta, F. S. Ling and P. Ramond, hep-ph/0306002; M. Bando, et al., hep-ph/0309310.

76. E. K. Akhmedov, M. Frigerio, A. Yu. Smirnov, JHEP 0309, 021 (2003).

77. W. Buchmuller, P. Di Bari and M. Plümacher, Nucl. Phys. B 643, 367 (2002).

78. A. Pilaftsis, T. E. J. Underwood, hep-ph/0309342.

79. B. Bajc, G. Senjanović, F. Vissani, Phys. Rev. Lett. 90, 051802 (2003).

80. T. Hambye, G. Senjanović, hep-ph/0307237.

81. H. S. Goh, R. N. Mohapatra and S-P. Ng, hep$\mathrm{ph} / 0303055$.
82. S. F. King, Phys. Lett. B 439, 350 (1998), Nucl. Phys. B 562, 57 (1999); S. Davidson and S. F. King, Phys. Lett. B 445, 191 (1998); for recent discussion see S. F. King, hep-ph/0310204.

83. K. S. Babu and S. M. Barr, Phys. Lett. B 381, 202 (1996); S. M. Barr, Phys. Rev. D 55, 1650 (1997). C. H. Albright and S. M. Barr, Phys. Rev. D 58, 013002 (1998); G. Altarelli and F. Feruglio, Phys. Lett. B 439, 112 (1998), JHEP 9811, 021 (1998).

84. K. S. Babu, C. N. Leung and J. Pantaleone, Phys. Lett. B 319, 191 (1993); J. R. Ellis and S. Lola, Phys. Lett. B 458, 310 (1999); N. Haba, et al., Eur. Phys. J. C10, 677 (1999); N. Haba, N. Okamura, M. Sugiura, Prog. Theor. Phys. 103, 367 (2000); J. A. Casas et al., Nucl. Phys. B 556, 3 (1999), ibidem, 569, 82 (2000), ibidem 573, 659 (2000); P.H. Chankowski, W. Krolikowski, S. Pokorski, Phys. Lett. B 472, 109 (2000); K. R. Balaji et al., Phys. Rev. Lett. 84, 5034 (2000); T. K. Kuo J. Pantaleone and G. H. Wu, Phys. Lett. B 518, 101 (2001); S. Antusch, M. Drees, J. Kersten, M. Lindner, M. Ratz, Phys. Lett. B 519, 238 (2001).

85. J. A. Casas, J. R. Espinosa and I. Navarro, hep$\mathrm{ph} / 0306243$.

86. S.T. Petcov, A.Yu. Smirnov, Phys. Lett. B 322, 109 (1994); A. S. Joshipura, Phys. Lett. B 543, 276 (2002); A. S. Joshipura, S. D. Rindani Phys. Rev. D 67, 073009 (2003), ibidem, Phys. Rev. D 67, 091302 (2003); S. Antusch, J. Kersten, M. Lindner, M. Ratz, hep-ph/0305273.

87. M. Fukugita and T. Yanagida, Phys. Lett. B 147, 45 (1986).

88. F. Vissani, A. Yu. Smirnov, Phys. Lett. B 341, 173 (1994). A. Brignole, H. Murayama, R. Rattazzi, Phys. Lett. B 335, 345 (1994).

89. M. Lindner, S. Antusch, J. Kersten, M. Lindner, M. Ratz, Phys. Lett. B 544, 1 (2002).

90. F. Borzumati and A. Masiero, Phys. Rev. Lett. 57, 961 (1986)

91. N. Arkani-Hamed, H-C. Cheng, J. L. Feng, L. J. Hall, Phys. Rev. Lett. 77, 1937 (1996).

92. I. Hinchliffe, F.E. Paige, Phys. Rev. D 63, 115006 (2001).

93. Y. Grossman, H. E. Haber, Phys. Rev. Lett. 78, 3438 (1997).

94. A. Romanino, A. Strumia, Nucl. Phys. B 622, 73 (2002); J. R. Ellis, J. Hisano, S. Lola (CERN), M. Raidal, Nucl. Phys. B 621, 208 (2002); for recent discussion see I. Masina, hep-ph/0304299.

95. S. Davidson, A. Ibarra, JHEP 0109, 013 (2001).

96. S. T. Petcov, et al., hep-ph/0306195.

97. A. Zee, Phys. Lett. B 93, 389 (1980), ibidem 161, 141 (1985);

98. For the latest discussion see, e.g. X. G. He hep$\mathrm{ph} / 0307172$.

99. K. S. Babu, Phys. Lett. B 203, 132 (1988).

100. L. J. Hall and M. Suzuki, Nucl. Phys. B 231, 419 (1984), A. Joshipura, M. Nowakowski, Phys. Rev. D 
51, 2421 (1995); A. Yu. Smirnov, F. Vissani, Nucl. Phys. B 460, 37 (1996); R. Hempfling, Nucl. Phys. B 478, 3 (1996); for the latest discussion see M. A. Diaz, et al., hep-ph/0302021.

101. N. Arkani-Hamed, S. Dimopoulos, G. R. Dvali and J. March-Russell, Phys. Rev. D 65, 02432 (2002); K. R. Dienes, E. Dudas and T. Ghergetta, Nucl. Phys. B 557, 25 (1999).

102. Y. Grossman and M. Neubert, Phys. Lett. B 474, 361 (2000).

103. G. R. Dvali, G. Gabadadze, M. Porrati, Phys. Lett. B 485, 208 (2000).

104. T. Appelquist and R. Shrock, Phys. Lett. B 548, 204 (2002); T. Appelquist, M. Piai, R. Shrock, hep$\mathrm{ph} / 0308061$.

105. F. Bazzocchi et al., hep-ph/0306184.

106. G. Seidl, hep-ph/0301044; K. S. Balaji, M. Lindner and G. Seidl, Phys. Rev. Lett. 91, 161803 (2003). 